How to cite this article: Adipose Tissue, Bï̈rntorp P, eJIFCC, vol 12 no 3, 2000: www.ifcc.org/ejifcc/vol12no3/adipose. htm

\section{Adipose tissue}

The adipose tissue is a diffuse organ whose main function is to store and give reserve energy in the form of triacylglycerol (triglycerides). Fat is stored in special cells, adipocytes, with specific functions. In this paper the adipose tissue will be discussed from the point of view of evolution, functional capacity, regional specificity and qualitative functions.

\section{Evolution}

Most species have some form of depot for the storage of extra energy. Some fishes, like the salmon, store their energy in the musculature while sharks store their energy in the liver. This can be practical as the energy is close to where it is needed. A draw back is that these depots are fairly limited. When larger amounts of energy need to be stored this is done in a special organ, the adipose tissue. Most mammals have their reserve energy stored in adipose tissue. The localisation of the adipose tissue shows great variation. Some rodents store their fat in the tail, perhaps they hope that an enemy should attack the tail instead of more vital parts of the body. The gorilla stores the fat in the neck and the upper part of the scull maybe in order to look more frightening. Some reindeers in the Arctic have their fat depots around the hoofs. Their fat is rich in unsaturated fatty acids and will protect them from extreme cold without solidifying. Seals are surrounded by a thick subcutaneous adipose depot that will insulate against the cold water.

Man has a subcutaneous as well as intra abdominal fat depots. The extremities are usually spared from the inconvenience of carrying subcutaneous fat. The big subcutaneous fat depots are found in the abdominal region and especially in women in the hip-thigh region. In these locations they hinder muscular function the least. The intra abdominal fat is found in the mesenterium and oment but also in the retroperitoneal region.

Sex differences in fat location are interesting and probably have a functional significance. Women have about $50 \%$ more adipose tissue than men probably due to their hip-thigh depots. The fat in these regions have a special function, it supplies the fetus during the latter part of gestation and also the new born child with energy. The fat from these depots is difficult to mobilise under ordinary conditions which is changed during the latter part of gestation and during lactation. Thus, this typical female adipose tissue localisation has also a specific female function. In a situation with adequate supply of food this function is not needed but is probably of great importance for the survival of the pregnant woman, fetus and new born child during times of varying food supply. One consequence of this specificity is that these depots are difficult to get rid of

\section{By Professor Per Björntorp,}

Department of internal medi-
cine, Sahlgrenska sjukhuset,
Göteborg, Sweden

Department of internal mediGöteborg, Sweden 
during attempts to reduce weight, an experience many women have made.

Men accumulate more of their depot fat in the visceral regions, i. e. the oment and mesenterium. This might also have a specific functional importance as these depots are drained to the vena porta and are easily mobilised. Prehistoric man, surviving through hunting and quick escape, had great need for easily mobilised energy that quickly reached the liver and its functions and entered the circulation as lipoproteins.

In obesity these depots are enlarged in a gender specific way, women accumulate their fat around thighs and hips ( back fatness) and men enlarge their depots centrally (belly fatness ). Sometimes one can see belly fatness in women but very seldom back fatness in men. It is the male belly fatness type that is associated with disease whereas back fatness is considerably less dangerous.

In our days, fat depots of a normal person contain energy for one month of total starvation. This was probably once of importance when the supply of energy was seasonal but of less importance when food is easily accessible. Long periods of starvation after years of bad harvests and long winters meant that thousands of people died. Those who survived without adequate external supply of energy were those who had large fat depots. It can well be that this meant genetic selection, they who could accumulate much fat survived. This could mean that we now have the selection of a so called thrifty gene, which might make the obesitas problem even more apparent. Could it be that the severe American obesitas problem could be caused by a selection of European obesity genes, those who had energy enough emigrated.

Other evolutionary mechanisms might also have contributed to the present obesitas epidemic. It is known that carbohydrate and protein intake is firmly regulated while the intake of fat is not in humans. Of course this had survival value, the easiest way to fill a reserve energy depot is to eat as much fat as is available, during a limited period of time. One can see this in other equivalent physiological phenomena. The main food of ice bears are seals. The ice bear can only catch the seals during winter. They are caught when they come up through the ice to breath. During summer they are very difficult to catch as they are much better swimmers than the ice bear. This means that the ice bear has magnificent depots of fat during winter time but is very lean and might even die of starvation during the summer.

The adipose tissue discussed so far is the so called white adipose tissue. There is also a so called brown adipose tissue which has the special function of producing heat. The oxidative phosphorylation is de-coupled and gives through a proton leakage in the mitochondria not ATP but heat. The main energy source is fat. Brown adipose tissue is found in many species and also in the new-born human.

Brown adipose tissue does probably not play a role in the pathogenesis of obesitas in humans. In Arctic species this is a necessary mechanism 
for survival. New-born seals have an effective way of their own of producing heat via the brown adipose tissue. Without an effectively protective fur and born on the ice they would not survive for long without this mechanism.

A dramatic example of the function of brown as well as white adipose tissue is lactation in certain seal species. The cubs are born on the ice near the open sea. They are threatened by carnivores and must as soon as possible get into the protective water. A condition for this is that the mother can quickly transfer enough energy to the cub through lactation to maintain enough endogenous heat production and also energy to make them ready to swim. This takes place with an astonishing speed and efficiency. During a few days several kilos of fat are transferred, after which the cubs are pushed into the protective water.

\section{Functional capacity}

The cells of the adipose tissue, the adipocytes, are formed from precursor cells in a multi-step differentiating process. The final product has all the specific functions of a adipocyte. The cell, with no fat accumulated, has a diameter of about 10-12 micrometers, about the same size as a lymphocyte. After normal fat accumulation the diameter has increased 10 -fold. This means that the volume has increased 1000-fold. It is well understood that the adipose tissue has an enormous plasticity and capacity to store depot energy.

The adipose tissue contains a number of other cell types. Endotelium cells are involved in the blood supply of the tissue while the function of other cells is not yet fully elucidated. The adipose tissue is richly enervated from the autonomous nervous system. Both the innervation and the blood-flow is of great importance to the function of the adipocytes.

\section{Regional specificity}

The regional specificity and its plausible physiological importance has been partly dealt with in an earlier section. In obesity all depots are more or less enlarged. A certain gender specific difference can be noted, men have more often abdominal fat and women more often back fatness but also abdominal fat. It is this latter type that is associated with disease. One can wonder why.

There are at present two types of hypotheses that try to explain this phenomenon. One hypothesis says that the central visceral fat depot generates risk factors for type 2 diabetes and cardio-vascular disease. The risk factors should according to this hypothesis be generated in the liver through the action of free fatty acids liberated from these easily mobilised abdominal fat depots. The production of lipoproteins is enhanced as the access to free fatty acids is speed limiting for their synthesis. The gluconeogenesis increases and results in increased glucose production. The uptake of insulin in the liver cells is prevented and a systemic hyperinsulinemia is created. The result is hyperlipoproteinemia, hypeinsulinemia and hyperglucemia all risk factors for diabetes and cardio-vascular 
disease. The weakness of this hypothesis is that it does not explain why the amount of visceral fat is increased. It is also apparent that the liver exposure of free fatty acids originating from the visceral fat is not dominating. The mass of the visceral fat is a small part of the total fat mass of the body and the liver cell exposure in all probability mainly comes from the systemic circulation via the arterial blood-flow of the liver.

An other hypothesis suggests that a central neuro-endocrine dysregulation exists in abdominal obesity. This is thought to direct fat to the visceral depots and also generate risk factors. Such a neuro-endocrine dysregulation works through activation of the hypothalamic-hypophysealadrenal axis (HPA-axis) resulting in periodically increased cortisol production. Cortisol is well known to direct depot fat to the visceral adipose tissue (e.g. Mb Cushing) and cause insulin resistance the two main symptoms in abdominal obesity. From the insulin resistance dyslipedimea can be derived and we have a full blown metabolic syndrome. Hypertonia which is often seen in connection with abdominal obesity is probably caused by a parallel activation of the central sympathetic nervous system which is strongly functionally connected with the HPA-axis. Different forms of stress my well be factors that activate these central regulating stations. This hypothesis has the advantage of encompassing the complete chain of different components. It is also possible that the two hypotheses can be combined and that both mechanisms contribute to the pathogenesis of the disease. This is an important and very active area of research.

\section{Qualitative functions}

The adipose tissue is a metabolically very active organ. The metabolic activity takes mainly place in the adipocytes. These cells have a rich set of hormonal receptors. These receptors are targets for hormones with both cell-surface and gene transcription functions.

The adipocytes have two principally different functions; they accumulate and they mobilise triacylglycerol. Storage of depot fat in the adipocytes is achieved through the caption of triacylglycerol rich particles by a capillary network of glucosaminoglucans. The triaclglycerols are subsequently hydrolysed by lipoprotein lipase. Fatty acids are captured by adjacent adipocytes presumably by membrane lateralisation, and then store in the fat globule of the cell. The lipoprotein lipase is synthesised in the adipocyte under the influence of a number of hormones, where insulin and cortisol are major stimulators. The insulin-cortisol interaction is starting a transcribtion effect of the lipoprotein lipase gene, and is also stabilising the enzyme by a posttranslational mechanism interfering with the dimerisation of the enzyme.

Fat metabolism is regulated primarily by another enzyme, the hormone sensitive lipase. This enzyme is activated directly by catecholamines mainly from the sympathetic nervous system via adrenergic receptors on the cell surface, leading to a cascade of events with phosphorylation of the lipase as the final activating step. Insulin is the major inhibitor. The lipase is the hydrolysing triacylglycerols resulting in the release of free fatty acids and glycerol. Some of the fatty acids are re-esterified in the 
adipocytes while others are released into the circulation where they are transported bound to albumin. These fatty acids are the major source of energy in the fasting state.

There are also hormones which amplify the catecholamine triggering of fat mobilisation. These are mainly steroid hormones

Recent research has shown that the adipocyte is also producing several other products than fatty acids and glycerol. Angiotensinogen and cytokines are examples of this. Adipose tissue also serves as a depot for steroid hormones.

Leptin is also produced in adipose tissue. Leptin is a peptide which regulates satiety via receptors in the hypothalamus. This is a useful mechanism from a physiological point of view, the adipose tissue is able to signal food intake via satiety regulation. The regulation is of a long term nature and the closer details are not yet known. Certain rodents have a mutation in the Leptin gene, other in the Leptin receptor gene.

The discovery of Leptin first gave hopes for a better understanding of human obesity. It turns out, however, that Leptin gene mutations are rare in human obesity, In contrast, obese humans as well as most obese animal models, except those with poor Leptin production due to mutations, show elevated Leptin concentrations in the circulation. This indicates a Leptin resistance. The nature of this resistance is currently subjected to intensive research.

\section{Summary}

Although adipose tissue was considered to be an inert tissue up till about 40 years ago, it has turned out that this is a very active tissue with a number of vital functions. Depot fat is stored here and mobilised in the postabsorptive phase, functions which are tightly regulated by hormones and the autonomous nervous system. Adipose tissue has also been shown to be a major store for fat soluble hormones as well as producer of signaling substances. In obesity adipose tissue is enlarged due to a positive energy balance. When this is occurring in central depots this is an index of risk for prevalent disease.
IFCC Technical Secretariat,

30 Rue Lionnois, F-54000 Nancy,

France

Phone: +33 $3 \quad 83 \quad 35 \quad 26 \quad 16$

Fax: $+\begin{array}{llllll}33 & 3 & 83 & 32 & 13 & 22\end{array}$

Email: Chantal.Thirion@ifccts.unancy.fr

Providing leadership in clinical chemistry and clinical laboratory service .

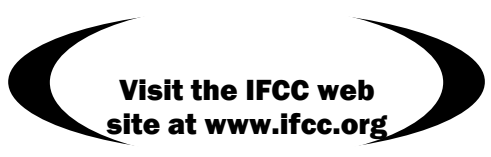

